\title{
CEP104 and CEP290; Genes with Ciliary Functions Cause Intellectual Disability in Multiple Families
}

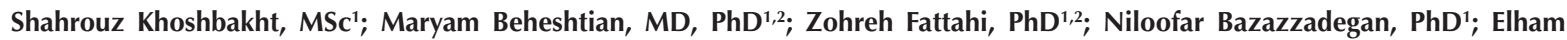 \\ Parsimehr, MSc ${ }^{2}$; Mahsa Fadaee, MSc ${ }^{2}$; Raheleh Vazehan, MSc ${ }^{2}$; Mehrshid Faraji Zonooz, MSc ${ }^{2}$; Ayda Abolhassani, MSc ${ }^{2}$; Mina \\ Makvand, MSc²; Ariana Kariminejad, MD²; Arzu Celik, PhD³; Kimia Kahrizi, MD; Hossein Najmabadi, PhD ${ }^{1,2^{*}}$ \\ ${ }^{1}$ Genetics Research Center, University of Social Welfare and Rehabilitation Sciences, Tehran, Iran \\ ${ }^{2}$ Kariminejad - Najmabadi Pathology \& Genetics Center, Tehran, Iran \\ 'Department of Molecular Biology and Genetics, Bogazici University, Istanbul, Turkey
}

\begin{abstract}
Background: Neurodevelopmental and intellectual impairments are extremely heterogeneous disorders caused by a diverse variety of genes involved in different molecular pathways and networks. Genetic alterations in cilia, highly-conserved organelles with sensorineural and signal transduction roles can compromise their proper functions and lead to so-called "ciliopathies" featuring intellectual disability (ID) or neurodevelopmental disorders as frequent clinical manifestations. Here, we report several Iranian families affected by ID and other ciliopathy-associated features carrying known and novel variants in two ciliary genes; CEP104 and CEP290.

Methods: Whole exome and targeted exome sequencing were carried out on affected individuals. Lymphoblastoid cell lines (LCLs) derived from the members of affected families were established for two families carrying CEP104 mutations. RNA and protein expression studies were carried out on these cells using qPCR and Western blot, respectively.

Results: A novel homozygous variant; NM_025114.3:c.7341_7344dupACTT p.(Ser2449Thrfs*8) and four previously reported homozygous variants; NM_025114.3:c.322C > T p.(Arg108*), NM_025114.3:c.4393C > Tp.(Arg1465*), NM_025114.3:c.5668G > T p.(Gly1890*) and NM_025114.3:c.1666dupA p.(Ile556Asnfs*20) were identified in CEP290. In two other families, two novel homozygous variants; NM_014704:c.2356_2357insTT p.(Cys786Phefs*11) and NM_014704:c.1901_1902insT p.(Leu634Phefs*33) were identified in CEP104, another ciliary gene. qPCR and Western blot analyses showed significantly lower levels of CEP104 transcripts and protein in patients compared to heterozygous or normal family members.

Conclusion: We emphasize the clinical variability and pleiotropic phenotypes due to the variants of these genes. In conclusion, our findings support the pivotal role of these genes in cognitive and neurodevelopmental features.

Keywords: CEP104, CEP290, Ciliopathies, Intellectual disability, Neurodevelopmental disorders

Cite this article as: Khoshbakht S, Beheshtian M, Fattahi Z, Bazazzadegan N, Parsimehr E, Fadaee M, et al. CEP104 and CEP290; genes with ciliary functions cause intellectual disability in multiple families. Arch Iran Med. 2021;24(5):364-373. doi: 10.34172/ aim.2021.53
\end{abstract}

Received: December 27, 2020, Accepted: March 13, 2021, ePublished: May 1, 2021

\section{Introduction}

Intellectual disability (ID) is considered a major health care problem causing a considerable socioeconomic burden on the population. ${ }^{1}$ ID can occur as part of neurological features in well-defined syndromes or it may present no other concomitant prominent symptoms, known as non-syndromic ID. ${ }^{2,3}$ ID shows an extreme level of genetic heterogeneity with many genes from virtually all functional molecular pathways and networks underlying the disorder. ${ }^{4-8}$ Genes responsible for ciliary functions, for example, are associated with clinical syndromes featuring ID as a key neurological outcome.

Cilia, microtubule-based organelles projecting from the cell membrane surface are found on a vast variety of eukaryotic cell types including most neuronal cells in the central nervous system. ${ }^{9}$ Non-motile (primary) cilia are thought to be involved in sensory functions (e.g. in olfactory neurons) and regulation of signal transduction. ${ }^{9-10}$ Defects in proteins localizing at cilia and centrosomes and/or contributing to ciliary and centrosomal functions are known to be associated with genetically heterogeneous and widely pleiotropic consequences known as "ciliopathies" with common neurological phenotypes. ${ }^{11-13}$ Since in the current study, we focus on the phenotypes of two ciliary genes, a brief introduction on their functions is given below;

\section{CEP104}

First identified as a centrosomal protein in a proteomic study of human centrioles, ${ }^{14} \mathrm{CEP} 104$ has been recently shown to be a key player in regulation of microtubule dynamics and ciliogenesis. ${ }^{15}$ During the cell cycle, CEP104 localizes to the distal ends of both mother and daughter centrioles and relocalizes from mother centriole to the tip of the elongating cilium when the cell exits from the cell cycle and starts to form cilia. ${ }^{16,17}$ This localization pattern is associated with interactions with the CEP97CP110 complex. ${ }^{17}$ Depletion of CEP104 results in 
compromised ciliogenesis or reduced-length cilia in RPE1 cells ${ }^{16,18}$ and its variants are associated with a ciliopathy disorder called Joubert syndrome (JBTS). ${ }^{19}$ In addition, its structure containing a canonical tubulin-binding, tumor overexpressed gene (TOG) domain provides more support for its role in ciliogenesis (Figure 1). ${ }^{15,18,20}$ A study in the multiciliated protozoan Tetrahymena using creation of knockout strains to investigate the localization and function of FAP256A (CEP104 ortholog in Tetrahymena) and two other ciliary proteins showed that FAP256A localizes at the boundary of distal segment and contributes to elongation of complete microtubules (A-tubules) by facilitating the addition of tubulin subunits to the ends of elongating microtubules. This study showed that FAP256A/CEP104 deficiency reduces the cilia parameters (cilia number and the length of A-tubules) and compromises the signaling functions of sensory cilia, which is the likely cause of JBTS. ${ }^{21}$ A high-throughput study trying to identify the interactome of human centrosomal and ciliary proteins using Proximity LabelMS showed extensive interaction of these proteins with dozens of partners including CEP104, CEP290 and other CEP proteins. ${ }^{22}$ CEP104 has been shown to interact with Nek1, which is involved in cilia stability ${ }^{15}$ and a recent study introduced the CEP104-CSPP1 complex as an important requisite for their proper function in regulating cilia length. Consistent with this result, Cep104-deficient zebrafish showed shortened cilia in Kupffer's vesicle together with cranial nerve abnormalities. ${ }^{23}$ Both CEP104 and CEP290 have been recently identified to be associated with a ciliary protein module containing ARMC9, TOGARAM1, CSPP1, RPGRIP1L and CCDC66 whose dysfunction can cause JBTS. ${ }^{24}$

\section{CEP290}

CEP290 was first identified as 3H11Ag, a tumor-associated antigen in different neoplastic cells. ${ }^{25}$ It was renamed as CEP290 after being recognized as a centrosomal protein through proteomic studies. ${ }^{26}$ This gene spans 54 exons and encodes a large multidomain protein with highly conserved motifs. ${ }^{27}$ CEP290 dynamically localizes to the centrosomes, nucleus, basal bodies, centriolar satellites and the transition zone in many different cells including photoreceptors. $^{28,29}$ Pathogenic variants of CEP290 have been linked to diverse ciliopathy phenotypes including leber congenital amaurosis (LCA), JBTS, Bardet-Biedl syndrome (BBS), Meckel syndrome and Senior-Loken syndrome. ${ }^{10,27-30}$ CEP290 contributes to interactions with many other centrosomal and ciliary proteins making it an important player for functions related to centrosome and cilia. CEP290 interacts with CCDC66 and PCM1 serving in ciliogenesis and ciliary trafficking. ${ }^{31}$ Together with PCM1, CEP290 is required for correct recruitment of RAB8A, a small GTPase, to the primary cilia where it exerts its ciliogenic effect. ${ }^{32} \mathrm{CEP} 290$ was also shown to colocalize and interact with $\mathrm{CC} 2 \mathrm{D} 2 \mathrm{~A}$, another ciliary protein linked to JBTS and related disorders and its knockdown synergistically exacerbates the pronephric cyst phenotype (ciliary dysfunction in zebrafish resembling human cystic kidney disease) in zebrafish sentinel phenotype. ${ }^{33}$

A

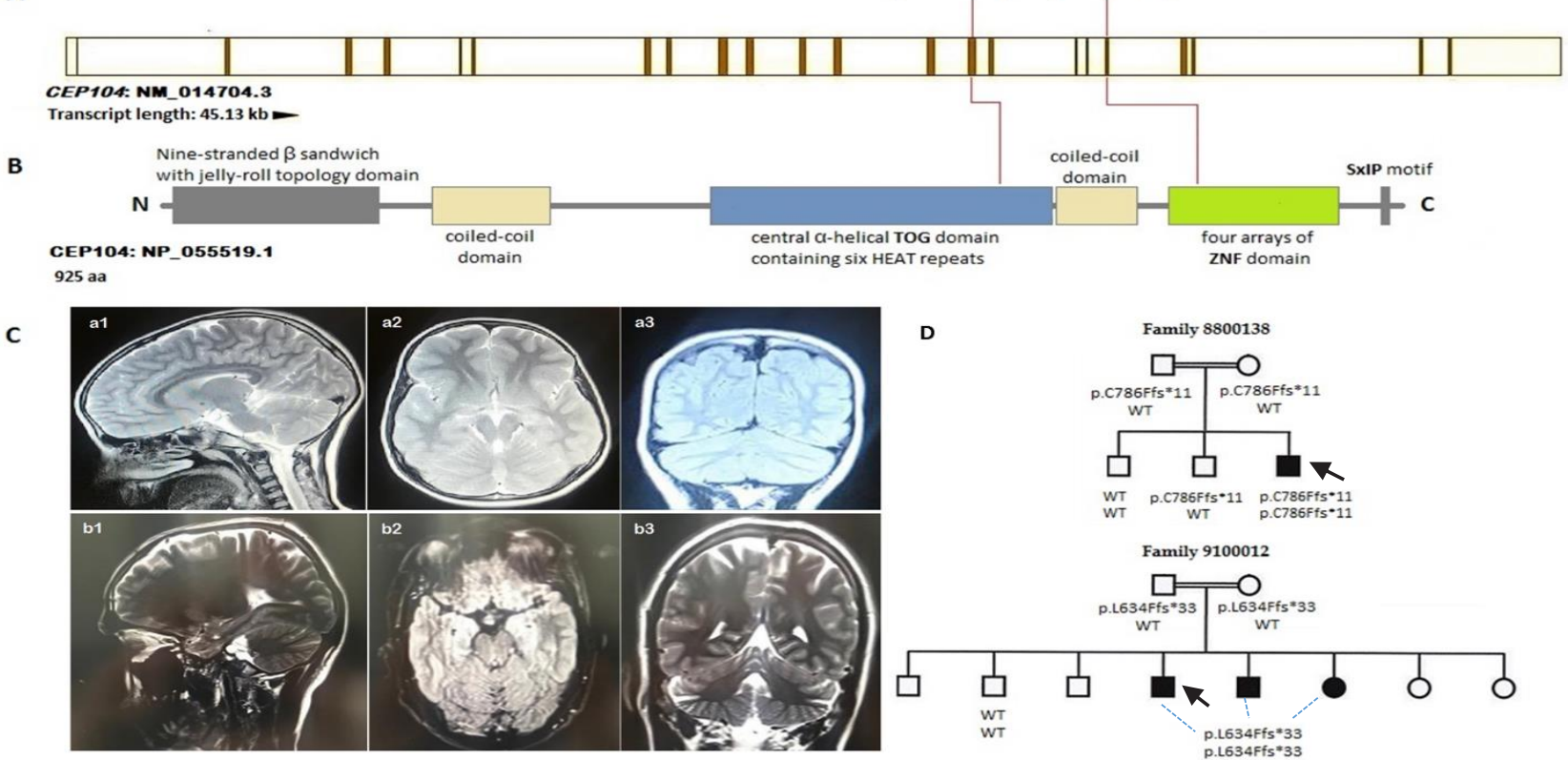

Figure 1. A) Schematic Representation of the Human CEP104. The exons are shown to scale as vertical bars. The mutations in families are shown relative to their positions in gene and protein domains. B) Schematic domain view of human CEP104, adapted from Al-Jassar et al. ${ }^{15}$ C) Axial, sagittal and flair views of brain MRI scans from patient in family 8800138 (a1, a2, a3) and a male patient from family $9100012(b 1, b 2, b 3)$ show normal brain structure with no characteristics of Joubert syndrome including molar tooth sign and hypoplasia of the cerebellar vermis. D) Pedigrees and segregation of the mutations within families. 
Among the other CEP290 partners are RPFR and ATF4, a microtubule-based transport protein and a cAMPdependent transcription factor involved in renal cyst development, respectively. ${ }^{28}$

As ID shows an extreme level of genetic heterogeneity, characterizing the genetic landscape in affected individuals could yield more knowledge on its molecular etiology and pathogenesis. Here, we report several families with previously reported and novel variants of two ciliary genes; CEP104 and CEP290 associated with syndromic and nonsyndromic forms of ID, adding evidence on the role of these genes in causing ID. The identified variants expand the genetic and phenotypic spectrum associated with these genes. We also aimed to elucidate the molecular effect of novel variants identified in CEP104 on transcriptome and protein expression to confirm their pathogenicity.

\section{Materials and Methods \\ Study Subjects}

Families recruited in this study were entirely from a largescale Next-generation sequencing (NGS) cohort of Iranian families affected with autosomal recessive intellectual disability (ARID) referred to the Genetics Research Center at the University of Social Welfare and Rehabilitation Sciences and Kariminejad - Najmabadi Pathology \& Genetics Center, Tehran, Iran between 2009 and 2018. Of those, families with identified causative variants in genes encoding centrosomal proteins (CEP) were chosen to be included in this study. A total of seven families were found to have such variants and enrolled in the study (see Table 1). In these families, either whole exome or targeted exome sequencing was used based on instructions from referring clinicians. All patients underwent detailed physical and clinical examination and clinical data were collected accordingly. Detailed phenotypic description of patients are provided in Supplementary file 1 and Table 1. The CEP104 variants in families 8800138 and 9100012 were novel variants previously reported by our group ${ }^{5}$ which have been further investigated by analyzing the variants' transcriptome and protein profiling in the current study.

\section{Genetic Analysis}

After obtaining informed consent, peripheral blood samples were taken from all affected members of the families. Genomic DNA was extracted using the saltingout method. Based on instructions from referring clinicians and clinical manifestations, either wholeexome or targeted sequencing was used, respectively for cases without or with phenotypic features specific to a given syndrome. The sequencing approach used for each family is shown in Table 1 and the list of genes for each targeted panel is provided in Supplementary Table S3. Exome enrichment of total genomic DNA was performed using Agilent Human All Exon kit (Agilent Technologies Inc., USA) and Illumina HiSeq sequencer (Illumina Inc., USA) was used for paired-end sequencing. Analysis of sequencing was carried out using Medical Resequencing
Analysis (MERAP) ${ }^{34}$ and Genome Analysis Toolkit $(\text { GATK })^{35}$ pipelines. Functional annotation of identified variants was performed using ANNOVAR ${ }^{36}$ tool. Neutral variants and previously reported variants in population databases with a frequency of $1 \%$ or above were filtered out from further analysis. Pathogenicity interpretation of identified variants was based on $\mathrm{ACMG}^{37}$ guidelines. The pathogenicity predictions and allele frequencies for variants identified in this study are provided in Supplementary Table S2. To ensure that identified mutations in patients are segregated in family members, Sanger sequencing was performed for family members when they were available for testing.

\section{Investigation of CEP104 RNA and Protein Expression in Lymphoblastoid Cell Lines}

In order to obtain a constant supply of biomolecules from study subjects, lymphoblastoid cell lines (LCLs) derived from the members of the affected families were established by in-vitro infection of peripheral blood B cells with Epstein-Barr virus using B95-8 lymphoblastoid cells. $^{38}$ RNA and protein expression studies were then carried out on these cells according to the manufacturer's instructions. To perform the transcriptome and protein level expression study, total cellular RNA and proteins were extracted from LCLs as described below. Following RNA extraction, using the RNeasy Mini Kit (Qiagen, Hilden, Germany) and concentration measurement (Nanodrop 2000 spectrophotometer, Thermo Scientific, Waltham, USA), cDNA synthesis was performed using a RevertAidFirst Strand cDNA Synthesis Kit (Thermo Scientific, Waltham, USA). Using specific primers for CEP104 (primer sequences are provided in Supplementary Table S1), qPCR based on SYBR green dye (SYBR Premix Ex Taq II, TAKARA, Japan) was performed to achieve the relative CEP104 expression in patients carrying the mutations compared to healthy family members genotyped as wild type or heterozygous for the same mutation (ABI 7500 instrument, Applied Biosystems, Foster City, USA). The relative expression for the GAPDH housekeeping gene was used to normalize the results and the instrument's ABI 7500 software 2.0.6 was employed to analyze the relative quantification data based on the $\Delta \Delta$ CT method.

We also aimed to carry out the protein expression level by Western blot analysis. To this end, the whole-cell proteins (cell lysates) from LCLs were isolated using RIPA (radioimmunoprecipitation assay) lysis buffer. The quality and concentration of extracted proteins were investigated via the Bradford assay (Quick Start, Bio-Rad Laboratories) by making a standard curve of serial dilutions of BSA protein using a NanoDrop device. Protein samples were electrophoresed on 10\% SDS-PAGE gels (Mini TransBlot Cell Module, Cat.1703810, Bio-Rad Laboratories) and then transferred to the PVDF membrane. To better evaluate the protein expression and distinguish between truncated short versions and NMD-undergone degraded 


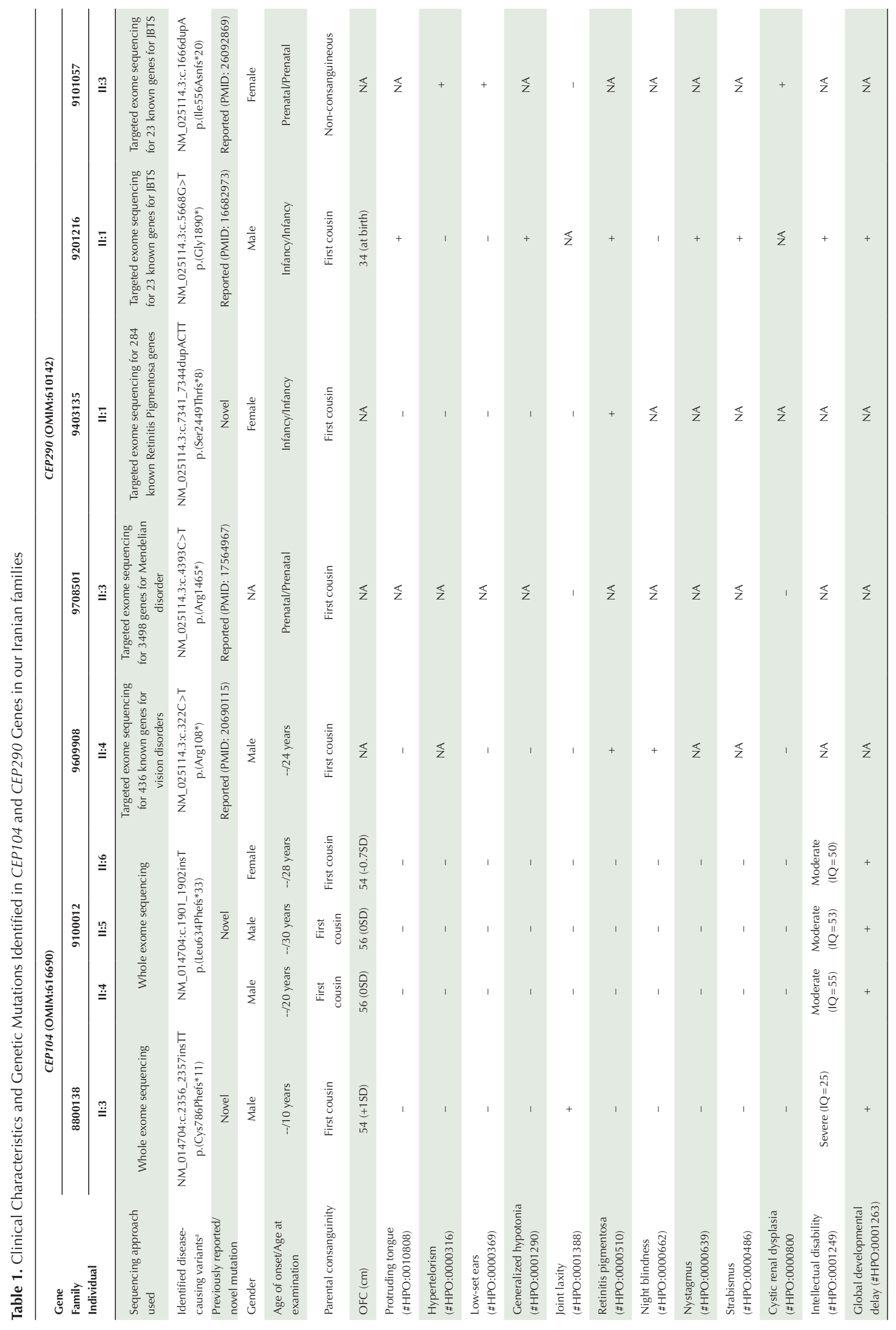




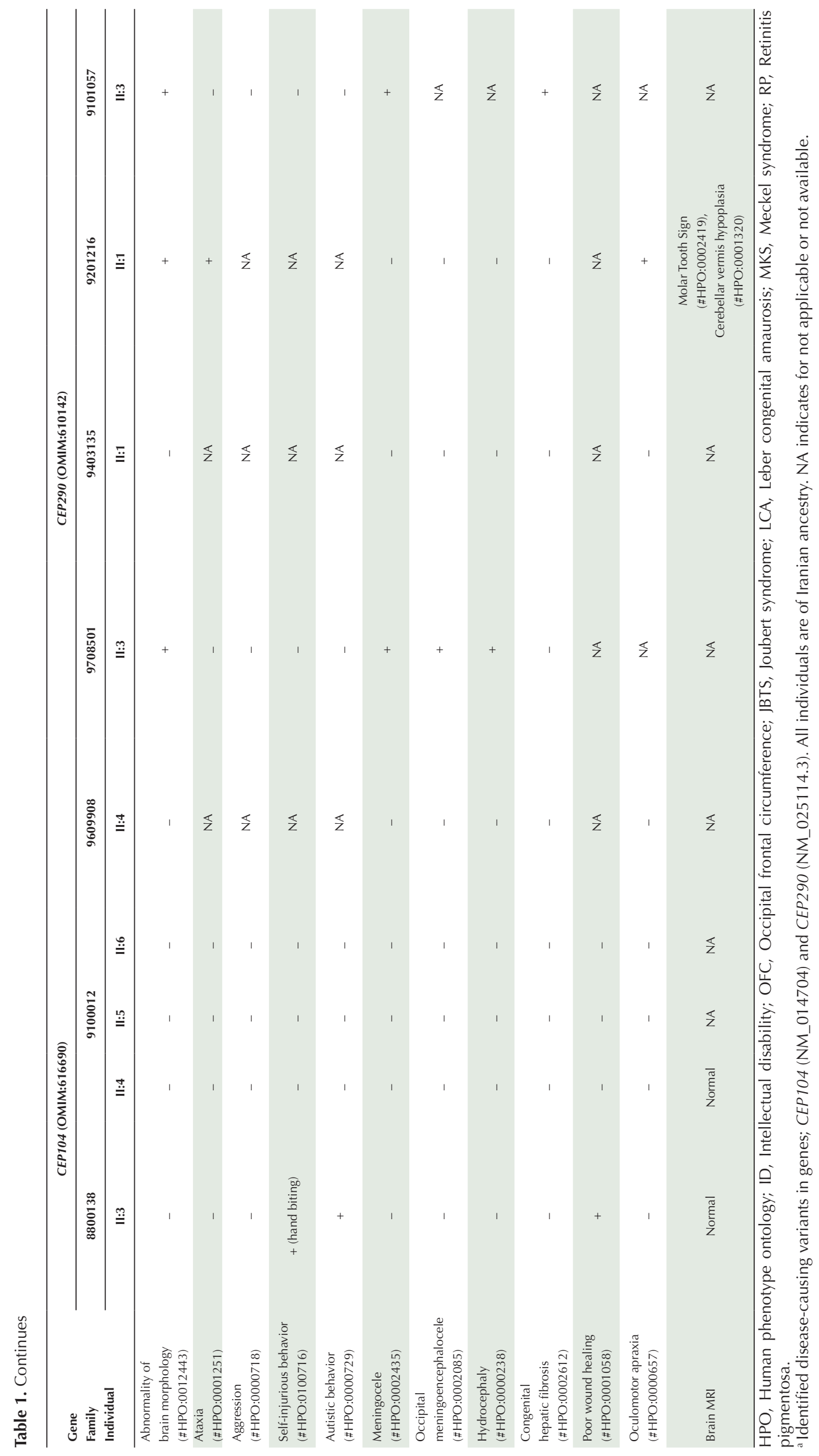


proteins, we used two primary antibodies for CEP104 protein, one against the N-terminus region (Santa Cruz, sc-515455) and the other for the region close to the C-terminus of the protein (Thermo Fisher Scientific, PA3101). Secondary antibodies used in this study included the Goat anti-Mouse (ab205719, Abcam) and Goat antiRabbit (ab205718, Abcam) HRP antibodies. The anti-beta Actin antibody (ab8226, Abcam) was used as loading control. The ECL Select Western blotting detection reagent (RPN2235, GE healthcare) was utilized to develop the bands.

\section{Results}

\section{Genetic Analysis Results}

We describe two and five families with causative variants of CEP104 and CEP290, respectively (pedigrees, identified variants and detailed clinical data are provided in Figures 1 and 2 and Table 1). In summary, we identified two novel homozygous variants in CEP104; NM_014704:c.2356_2357insTT p.(Cys786Phefs $\left.{ }^{\star} 11\right)$ and NM_014704:c.1901_1902insT p.(Leu634Phefs $\left.{ }^{\star} 33\right)$ in the affected members of two families. In the remaining five families, a novel homozygous variant; NM_025114.3:c.7341_7344dupACTT p.(Ser2449Thrfs $\left.{ }^{\star} 8\right)$ and four previously reported homozygous variants; NM_025114.3:c.322C > T p. $\left(\operatorname{Arg} 108^{\star}\right), \quad$ NM_025114.3:c.4393C $>$ T p. $\left(\operatorname{Arg} 1465^{\star}\right)$, NM_025114.3:c.5668G > T p. $($ Gly1890*) and NM_025114.3:c.1666dupA p.(Ile556Asnfs $\left.{ }^{\star} 20\right)$ were identified in CEP290. As mentioned, all the variants were homozygous and were confirmed by Sanger sequencing. The variants were found to be segregated in the family members compatible with recessive inheritance. However, for families 9609908 and 9403135, the heterozygous variants in the parents were not confirmed due to the unavailability of samples. For the family 9101057, as none of the aborted fetuses were available for sequencing, the causative homozygous NM_025114.3:c.1666dupA p.(Ile556Asnfs $\left.{ }^{\star} 20\right)$ variant in the fetuses was not confirmed (Figures 1D and 2C). Our patients with CEP104 variants, family 8800138; NM_014704:c.2356_2357insTT p.(Cys786Phefs $\left.{ }^{\star} 11\right)$ and family 9100012; NM_014704:c.1901_1902insT p.(Leu634Phefs ${ }^{\star 33)}$ showed non-syndromic ID with no brain malformations such as cerebellar hypoplasia or pathognomonic molar tooth sign (MTS) (Figure 1C), previously reported for patients with JBTS carrying CEP104 mutations. ${ }^{19,39}$ This may indicate that at least in some cases, CEP104 is less likely to cause brain malformations; rather, it can possibly affect higher cognitive and intellectual capabilities. Our patients with CEP290 variants manifested an extensive phenotypic spectrum compatible with known phenotypes related to CEP290 variants. Clinical findings in the family 9403135; NM_025114.3:c.7341_7344dupACTT p.(Ser2449Thrfs $\left.{ }^{\star} 8\right)$ showing LCA and the family 9609908 ; NM_025114.3:c.322C > T p. $\left(\operatorname{Arg} 108^{\star}\right)$ presenting with night blindness and hearing loss were in line with CEP290 localization and function in the cilia of photoreceptor cells. ${ }^{28}$ The proband in the family 9201216 ; NM_025114.3:c.5668G > T p. $($ Gly1890*) was referred with LCA and typical symptoms associated with JBTS, including MTS and developmental delay. The aborted fetuses in families 9708501; NM_025114.3:c.4393C > T p.(Arg1465*) and 9101057; NM_025114.3:c.1666dupA

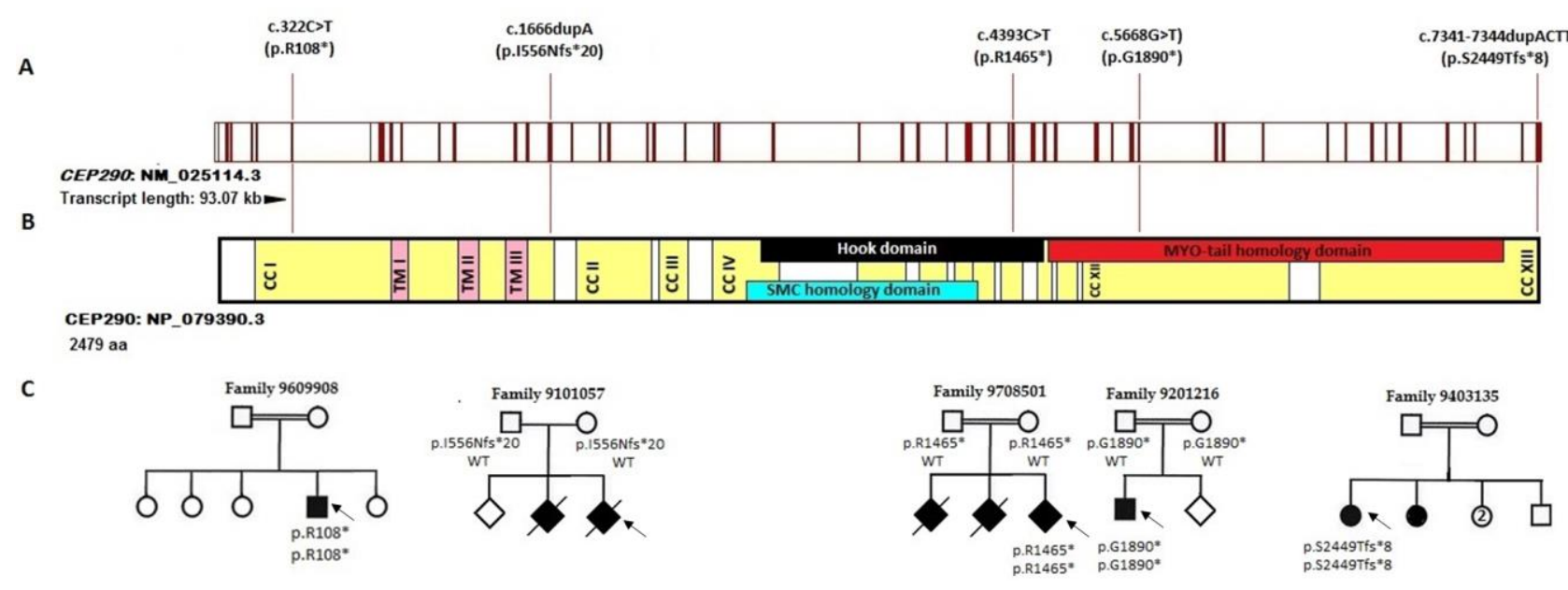

Figure 2. A) Schematic Representation of the Human CEP290. The exons are shown to scale as vertical bars. The mutations in families are shown relative to their positions in gene and protein domains. B) Schematic domain view of human CEP290 protein, adapted from Sayer et al. (2006) and Moradi et al. (2011). A 13 interspaced series of coiled-coil domains constitute the main structure of CEP290. There are other motifs between coiled-coil domains, including KID, P-loop and BP-NLS motifs (not shown). C) Pedigrees and segregation of the mutations within families. For families 9708501 and 9201216, cosegregation of the variants was confirmed by sequencing. In families 9609908 and 9403135, the heterozygous variants in the parents were not confirmed due to the unavailability of samples. For family 9101057 , the causative homozygous variant in the fetuses (in heterozygous status in parents) were not confirmed due to unavailability of aborted fetuses for sequencing. Abbreviations: CC: coiled-coil domain, MYO-tail: myosin-tail homology domain, SMC: structural maintenance of chromosomes (SMC) chromosome segregation ATPase homology domain , TM: tropomyosin homology domain, WT: wild-type. 
p.(Ile556Asnfs $\left.{ }^{\star} 20\right)$ showed clinical manifestations linked to Meckel-Gruber syndrome. All our patients except for family 9101057 were born to consanguineous families and the inheritance pattern in all pedigrees was compatible with the recessive mode which is in line with a putative inheritance mode for most ciliopathy genes.

\section{RNA and Protein Expression Profiles of Patient-Derived Lymphoblastoid Cells}

We established LCLs for all healthy and affected members of the families 8800138 and 9100012 carrying truncating variants in CEP104. The effects of the variants on the RNA and protein expression levels were then investigated in these cells using qPCR and Western blot analysis, respectively. Quantitative analysis of the CEP104 mRNA expression level revealed a significant reduction of CEP104 transcripts in the patients' cells compared to the unaffected wild-type and heterozygous members of the family (Figure 3A).

Weak traces of protein expression in homozygous patients with truncating variants were detected using CEP104 primary antibody specific to the $\mathrm{N}$-terminus region of the protein. However, repeated experiment using the C-terminus-binding CEP104 antibody did not reveal specific bands indicating the absence of the respective epitope in affected individuals (Figure 3B). This finding shows the lower expression of the truncated protein rather than complete nonsense-mediated decay (NMD) in patients carrying truncating variants.

\section{Discussion}

CEP (centrosomal) proteins are involved in ciliary functions and in the regulation of cell division, motility and signal transduction through their complex interaction networks with other ciliary proteins, especially with major ciliopathy proteins showing neurological consequences. ${ }^{10,21}$ CEP104 is a ciliary protein localizing at primary cilia which play central roles in transducing or regulating several signaling pathways; defects in primary cilia contribute to a group of disorders known as "ciliopathies" that can adversely affect development of the brain and other essential organs. ${ }^{11-13}$ Previously reported patients with CEP104 variants $^{19,35}$ all manifested typical JBTS with neurologic manifestations including MTS, oculomotor apraxia and hypotonia alongside significant developmental delay with the absence of polydactyly, liver or renal involvement. Our patients, however, did not show typical JBTS; rather, they manifested ID with no attributable syndromes. The patient in family 8800138; NM_014704:c.2356_2357insTT p.(Cys786Phefs $\left.{ }^{\star} 11\right)$ also showed joint laxity, stereotypic hand movement and autistic phenotype. MRI imaging did not show JBTS-

A
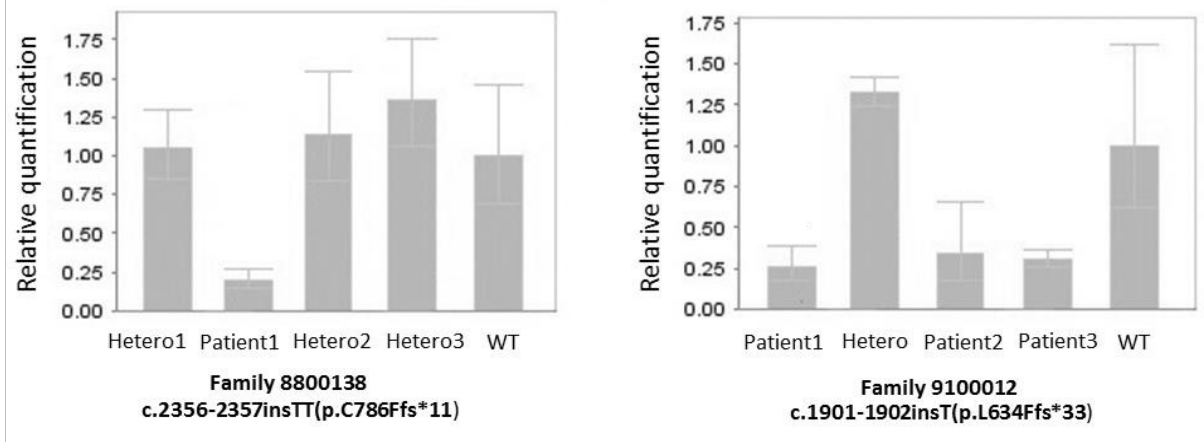

B

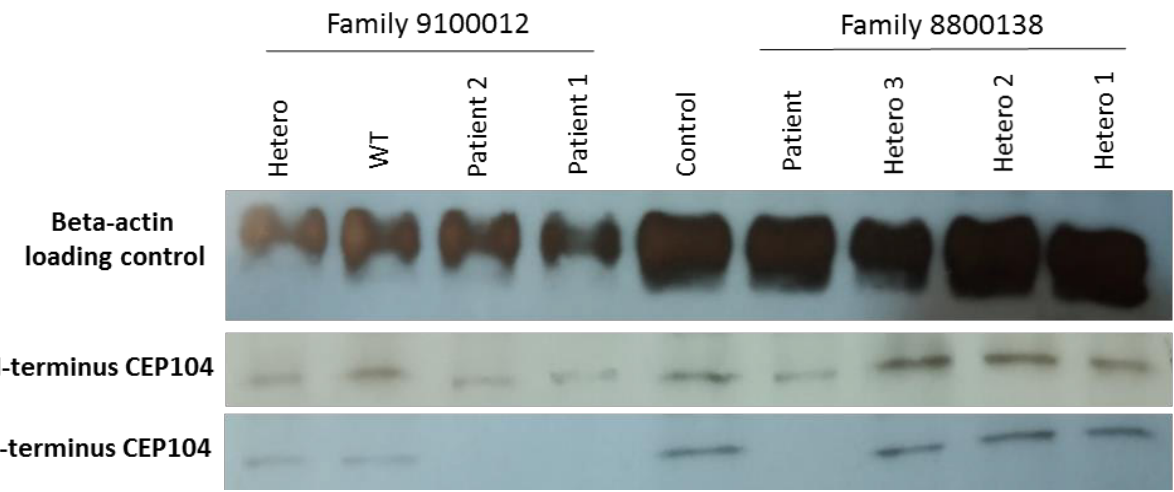

Figure 3. A) The qPCR Analysis of CEP104 Transcripts in Families 8800138 and 9100012 . The results indicate a significant reduction of transcripts in patients compared to other family members. Each bar represents a single individual. The results were normalized by comparison to the GAPDH expression level. B) Western blot analysis to detect CEP104 protein in whole-cell lysates from lymphoblastoid cell lines. The order of loaded samples indicated for the first blot is the same for all three blots. Patients in both families show weak expression using the CEP104 N-terminus antibody whereas no protein expression is seen in their profiles using a C-terminus antibody indicating the lower-level expression of truncated CEP104 in patients (Hetero: Heterozygote, WT: Wild-type). 
related neurologic signs. The family 9100012 patients; NM_014704:c.1901_1902insT p.(Leu634Phefs ${ }^{\star 33)}$ showed merely ID with no defective MRI imaging or skeletal disorders. The locations of variants in these two families are conserved and lie in functional domains (zinc finger and TOG domains, respectively) so that their deleterious effects are not unlikely. Taken together, the incomplete overlap of the phenotypic spectrum between previously reported patients carrying CEP104 mutations and that of our patients may be explained by the extensive phenotypic spectrum observed in ciliopathies affecting almost every organ. In addition, ciliopathies represent genetic and allelic heterogeneity. ${ }^{24}$ In the case of JBTS, for instance, 34 genes have been linked to the disorder to date (http://www.omim.org/). Furthermore, the allelic heterogeneity of ciliopathy genes increases the heterogeneity linked to the ciliopathies. ${ }^{19}$ Therefore, our study expands the mutational and clinical phenotypes reported for CEP104, which may have implications for probable genotype-phenotype correlations as well as understanding its molecular function.

Given the increasing number of proteins contributing to interactions with CEP290, it is not unlikely that it serves as a key hub in ciliary/centrosomal molecular networks. Centrosomal and ciliary proteins share multiple interactions in response to extracellular signaling associated with the homeostasis of tissues and neuronal development. ${ }^{40}$ This may explain the overlapping yet distinct ciliopathy phenotypic spectrum resulting from CEP290 pathogenic variants, defects of which may leave retinal, renal and neuronal phenotypes including ID as major consequences. As an example, through its interaction with RAB8A which is in direct collaboration with the BBSome complex leading to centrosomal localization of BBS4, CEP290 makes a bridge with this complex broadening its potential interaction network. ${ }^{32}$ Since CEP290 is implicated in the BBSome molecular network linked to BBS, it is not surprising that CEP290 variants cause diverse phenotypic outcomes conferring a clue to unravel molecular mechanisms and genetic links underlying ciliopathies. Consistent with the heterogeneity and pleiotropy of phenotypes associated with CEP290 causative variants previously reported in other studies, ${ }^{27-29,41}$ our families with CEP290 variants showed various phenotypes.

Taken together, the extensive phenotypic spectrum in our patients further underscores the importance of CEP genes in primary cilia, defects of which could result in highly pleiotropic phenotypes including neurodevelopmental signs (as seen in the aborted fetuses of families 97018501 and 9101057 with CEP290 variants) and ID (family 9201216 with CEP290 variants and families 8800138 and 9100012 carrying CEP104 variants).

In conclusion, identification of new variants in genes previously implicated in ciliopathies such as CEP104 and CEP290 can expand the phenotypic spectrum associated with these disorders and may even establish some genotype-phenotype correlations. Similarly, identification of novel genes linked to ciliopathies may further elucidate the molecular mechanisms underlying these disorders. In addition, it yields more implications for genetic counseling and screening for families affected with these disorders.

\section{Authors' Contribution}

SK carried out the experiments and wrote the drafting manuscript. MB, ZF and AK supervised the analysis of NGS data. NB substantially provided technical support and contributed to data analysis. EP, MF, RV, MFZ, AA, and MM were involved in NGS data collation and analysis. AC provided scientific support and edited the final manuscript. KK and HN critically reviewed and revised the intellectual content of the manuscript. KK also described the patients' phenotypes and approved their final diagnosis. $\mathrm{HN}$ also conceived and designed the whole project.

\section{Conflict of Interest Disclosures}

The authors declare no conflict of interest.

\section{Ethical Statement}

This study was approved by the Ethics Committee of the University of Social Welfare and Rehabilitation Sciences and all participants or their legal representatives signed written informed consent for participation in the study.

\section{Data Availability Statement}

The data supporting our findings for families 9101057, 9201216, 9403135, 9708501 and 9609908 are openly available in the ClinVar database at https://submit.ncbi.nlm.nih.gov/clinvar/ under submission IDs: SUB6694481 and SUB7121713. For families 8800138 and 9100012 (doi: 10.1038/s41380-017-00122 ), data sharing is not applicable as no new data were created or analyzed in this study.

\section{Acknowledgments}

We express our sincere thanks to the families who participated in this study and their genuine collaboration. This research was financially supported by the National Institute for Medical Research Development (NIMAD) (grant nos.: 958715 to HN and 957060 to KK), Iran National Science Foundation (INSF) (grant no. 950022 to HN), and University of Social Welfare and Rehabilitation Sciences (grant no. 98/801/A/6/2492 to HN).

\section{Supplementary Materials}

Supplementary file 1 contains detailed clinical description and Tables S1-S2. Supplementary file 2 contains Table S3.

\section{References}

1. Musante H, Ropers HH. Genetics of recessive cognitive disorders. Trends Genet. 2014;30(1):32-39. doi: 10.1016/j. tig.2013.09.008

2. Vissers LE, Gillisen C, Veltman JA. Genetic studies in intellectual disability and related disorders. Nat Rev Genet. 2016;17(1):9-18. doi: 10.1038/nrg3999

3. Kaufman L, Ayub M, Vincent JB. The genetic basis of nonsyndromic intellectual disability: a review. J Neurodev Disord. 2010; 2(4):182-209. doi: 10.1007/s11689-010-90552

4. Hu H, Kahrizi K, Musante L, Fattahi Z, Herwig R, Hosseini $\mathrm{M}$, et al. Genetics of intellectual disability in consanguineous 
families. Mol Psychiatry. 2019; 24(7):1027-39. doi: 10.1038/ s41380-017-0012-2

5. Najmabadi H, Hu H, Garshasbi M, Zemojtel T, Abedini SS, Chen W, et al. Deep sequencing reveals 50 novel genes for recessive cognitive disorders. Nature. 2011;478(7367):5763. doi: 10.1038/nature 10423

6. Kochinke K, Zweier C, Nijhof B, Fenckova M, Cizek P, Honti F, et al. Systematic phenomics analysis deconvolutes genes mutated in intellectual disability into biologically coherent modules. Am J Hum Genet. 2016;98(1):149-64. doi: 10.1016/j.ajhg.2015.11.024

7. Ataei R, Khoshbakht S, Beheshtian M, Abedini SS, Behravan H, Esmaeili Dizghandi S, et al. Contribution of Iran in elucidating the genetic causes of autosomal recessive intellectual disability. Arch Iran Med. 2019;22(8):461-71.

8. Rauch A, Wieczorek D, Graf E, Wieland T, Endele $S$, Schwarzmayr $\mathrm{T}$, et al. Range of genetic mutations associated with severe non-syndromic sporadic intellectual disability: an exome sequencing study. Lancet. 2012;10;380(9854):1674-82. doi: 10.1016/S01406736(12)61480-9.

9. Lee JE, Gleeson JG. A systems-biology approach to understanding the ciliopathy disorders. Genome Med. 2011;3(9):59. doi: 10.1186/gm275

10. Reiter JF, Leroux MR. Genes and molecular pathways underpinning ciliopathies. Nat Rev Mol Cell Biol. 2017;18(9):533-47. doi: 10.1038/nrm.2017.60

11. Travaglini L, Brancati F, Attie-Bitach T, Audollent S, Bertini E, Kaplan J, et al. Expanding CEP290 mutational spectrum in ciliopathies. Am J Med Genet A. 2009;149A(10):2173-80. doi: 10.1002/ajmg.a.33025

12. Srour M, Schwartzentruber J, Hamdan FF, Ospina LH, Patry L, Labuda D, et al. Mutations in C5ORF42 cause Joubert syndrome in the French Canadian population. Am J Hum Genet. 2012;90(4):693-700. doi: 10.1016/j.ajhg.2012.02.011

13. Wheway G, Mitchison HM. Genomics England Research Consortium. Opportunities and challenges for molecular understanding of ciliopathies-the 100,000 genomes project. Front Genet. 2019;11;10:127. doi: 10.3389/ fgene.2019.00127. Erratum in: Front Genet. 2019 Aug $13 ; 10: 569$.

14. Jakobsen L, Vanselow K, Skogs M, Toyoda Y, Lundberg E, Poser I, et al. Novel asymmetrically localizing components of human centrosomes identified by complementary proteomics methods. EMBO J. 2011;30(8):1520-35. doi: 10.1038/emboj.2011.63

15. Al-Jassar C, Andreeva A, Barnabas DD, McLaughlin SH, Johnson CM, Yu M, et al. The ciliopathy-associated Cep104 protein interacts with tubulin and Nek1 kinase. Structure. 2017;25(1):146-56. doi: 10.1016/j.str.2016.11.014

16. Satish Tammana TV, Tammana D, Diener DR, Rosenbaum J. Centrosomal protein CEP104 (Chlamydomonas FAP256) moves to the ciliary tip during ciliary assembly. J Cell Sci. 2013; 126(Pt21):5018-29. doi: 10.1242/jcs.133439

17. Rezabkova L, Kraatz SH, Akhmanova A, Steinmetz MO, Kammerer RA. Biophysical and structural characterization of the centriolar protein Cep104 interaction network. J Biol Chem. 2016;291(35):18496-504. doi: 10.1074/jbc. M116.739771

18. Jiang K, Toedt G, Montenegro Gouveia S, Davey NE, Hua S, van der Vaart B, et al. A proteome wide-screen for mammalian SxIP motif-containing microtubule plus-end tracking proteins. Curr Biol. 2012;22(19):1800-807. doi: 10.1016/j.cub.2012.07.047

19. Srour M, Hamdan FF, McKnight D, Davis E, Mandel H, Schwartzentruber J, et al. Joubert syndrome in French Canadians and identification of mutations in CEP104. Am J Hum Genet. 2015;97(5):744-53. doi: 10.1016/j. ajhg.2015.09.009

20. Slep KC. A cytoskeletal symphony: Owed to TOG. Dev Cell. 2018;46(1):5-7. doi: 10.1016/j.devcel.2018.06.010

21. Louka P, Vasudevan KK, Guha M, Joachimiak E, Wloga D, Tomasi RF, et al. Proteins that control the geometry of microtubules at the ends of cilia. J Cell Biol. 2018;217(12):4298-313. doi: 10.1083/jcb.201804141

22. Gupta GD, Coyaud E, Goncalves J, Mojarad BA, Liu Y, Wu Q, et al. A Dynamic Protein Interaction Landscape of the Human Centrosome-Cilium Interface. Cell. 2015;163(6):1484-99. doi: 10.1016/j.cell.2015.10.065

23. Frikstad KM, Molinari E, Thoresen M, Ramsbottom SA, Hughes F, Letteboer SJF, et al. A CEP104-CSPP1 complex is required for formation of primary cilia competent in Hedgehog signaling. Cell Rep. 2019;28(7):1907-22.e6. doi: 10.1016/j.celrep.2019.07.025

24. Latour BL, Van De Weghe JC, Rusterholz TD, Letteboer SJ, Gomez A, Shaheen R, et al. Dysfunction of the ciliary ARMC9/TOGARAM1 protein module causes Joubert syndrome. J Clin Invest. 2020;130(8):4423-39. doi: 10.1172/ JCI131656.

25. Chen D, Shou C. Molecular cloning of tumor-associated antigen recognized by monoclonal antibody $3 \mathrm{H} 11$. Biochem Biophys Res Commun. 2001;280(1):99-103. doi: 10.1006/bbrc.2000.4087

26. Andersen JS, Wilkinson CJ, Mayor T, Mortensen P, Nigg EA, Mann M. Proteomic characterization of human centrosome by protein correlation profiling. Nature. 2003;426(6966):570-4. doi: 10.1038/nature.02166

27. Moradi P, Davies WL, Mackay DS, Cheetham ME, Moore AT. Focus on molecules: centrosomal protein 290 (CEP290). Exp Eye Res. 2011;92(5):316-7. doi: 10.1016/j. exer.2010.05.009

28. Sayer JA, Otto EA, O’Toole JF, Nurnberg G, Kennedy MA, Becker $\mathrm{C}$, et al. The centrosomal protein nephrocystin- 6 is mutated in Joubert syndrome and activates transcription factor ATF4. Nat Genet. 2006;38(6):674-81. doi: 10.1038/ ng1786

29. Sheck L, Davies WIL, Moradi P, Robson AG, Kumaran N, Liasis AC, et al. Leber Congenital Amaurosis Associated with Mutations in CEP290, Clinical Phenotype, and Natural History in Preparation for Trials of Novel Therapies. Ophthalmology. 2018;125(6):894-903. doi: 10.1016/j. ophtha.2017.12.013

30. Valente EM, Silhavy JL, Brancati F, Barrano G, Krishnaswami SR, Castori M, et al. Mutations in CEP290, which encodes a centrosomal protein, cause pleiotropic forms of Joubert syndrome. Nat Genet. 2006;38(6):623-5. doi: $10.1038 /$ ng1805

31. Conkar D, Culfa E, Odabasi E, Rauniyar N, Yates JR 3rd, Firat-Karalar EN. The centriolar satellite protein CCDC66 interacts with CEP290 and functions in cilium formation and trafficking. J Cell Sci. 2017;130(8):1450-62. doi: $10.1242 /$ jcs. 196832

32. Kim J, Krishnaswami SR, Gleeson JG. CEP290 interacts with the centriolar satellite component PCM-1 and is 
required for Rab8 localization to the primary cilium. Hum Mol Genet. 2008;17(23):3796-805. doi: 10.1093/hmg/ $\operatorname{ddn} 277$

33. Gorden NT, Arts HH, Parisi MA, Coene KL, Letteboer SJ, van Beersum SE, et al. CC2D2A is mutated in Joubert syndrome and interacts with the ciliopathy-associated basal body protein CEP290. Am J Hum Genet. 2008;83(5):55971. doi: $10.1016 /$ j.ajhg.2008.10.002

34. Hu H, Wienker TF, Musante L, Kalscheuer VM, Kahrizi K, Najmabadi $\mathrm{H}$, et al. Integrated sequence analysis pipeline provides one-stop solution for identifying disease-causing mutations. Hum Mutat. 2014;35(12):1427-35. doi: 10.1002/ humu.22695.

35. McKenna A, Hanna M, Banks E, Sivachenko A, Cibulskis K, Kernytsky A, et al. The Genome Analysis Toolkit: a MapReduce framework for analyzing next-generation DNA sequencing data. Genome Res. 2010 Sep;20(9):1297303. doi: 10.1101/gr.107524.110.

36. Wang K, Li M, Hakonarson H. ANNOVAR: functional annotation of genetic variants from high-throughput sequencing data. Nucleic Acids Res. 2010 Sep;38(16):e164. doi: 10.1093/nar/gkq603. Epub 2010 Jul 3.

37. Richards S, Aziz N, Bale S, Bick D, Das S, Gastier-Foster
J, et al. ACMG Laboratory Quality Assurance Committee. Standards and guidelines for the interpretation of sequence variants: a joint consensus recommendation of the American College of Medical Genetics and Genomics and the Association for Molecular Pathology. Genet Med. 2015;17(5):405-24. doi: 10.1038/gim.2015.30.

38. Hussain T, Mulherkar R. Lymphoblastoid cell lines: a continuous in vitro source of cells to study carcinogen sensitivity and DNA repair. Int J Mol Cell Med. 2012;2(1):7587

39. Luo M, Cao L, Cao Z, Ma S, Shen Y, Yang D, et al. Whole exome sequencing reveals novel CEP104 mutations in a Chinese patient with Joubert syndrome. Mol Genet Genomic Med. 2019;7(12):e1004. doi: 10.1002/mgg3.1004

40. Badano JL, Teslovich TM, Katsanis N. The centrosome in human genetic disease. Nat Rev Genet. 2005;6(3):194-205. doi: 10.1038/nrg.1557

41. Bachmann-Gagescu R, Dempsey JC, Phelps IG, O’Roak BJ, Knutzen DM, Rue TC, et al. Joubert syndrome: a model for untangling recessive disorders with extreme genetic heterogeneity. J Med Genet. 2015;52(8):514-22. doi: 10.1136/jmedgenet-2015-103087 